\section{Información, conocimiento y percepción sobre el riesgo de contraer el dengue en Argentina: dos experiencias de intervención para generar estrategias locales de control}

\author{
Information, knowledge, and perception of dengue \\ risk in Argentina: two intervention experiments \\ to generate local control strategies
}

Nicolas Schweigmann 1,2

Andrea Rizzotti 3

Gabriela Castiglia 4

Fabio Gribaudo 4

Edgardo Marcos 5

Nora Burroni 1

Gabriela Freire 1

Vanesa D'Onofrio ${ }^{1}$

Sara Oberlander 1

Héctor Schillaci 1

Sandra Gómez 1

Santiago Maldonado 1

Claudia Serrano 1

\footnotetext{
${ }^{1}$ Facultad de Ciencias Exactas y Naturales, Universidad de Buenos Aires, Buenos Aires, Argentina. 2 Consejo Nacional de Investigaciones Científicas y Técnicas, Ministerio de Ciencia, Tecnología e Innovación Productiva Buenos Aires, Argentina. ${ }^{3}$ Facultad Latinoamericana de Ciencias Sociales, Buenos Aires, Argentina. 4 Secretaría de Salud Pública del Municipio de Vicente López, Vicente López, Argentina.

5 Instituto de Zoonosis Luis Pasteur, Buenos Aires, Argentina.

Correspondencia N. Schweigmann Departamento de Ecología, Genética y Evolución Facultad de Ciencias Exactas y Naturales, Universidad de Buenos Aires.

Intendente Güiraldes $s / n$, Pabellón II, Cuarto piso, Laboratorio 52, Ciudad Universitaria, Buenos Aires C1428EHA, Argentina. nicolas@ege.fcen.uba.ar
}

\begin{abstract}
With the objectives of dengue prevention, health promotion, and action-based research, two experiments were conducted in pilot areas of Buenos Aires and Vicente López, Argentina. In each area, community, government, and non-governmental references were identified. The study included an entomological survey (with ovitraps), environmental survey (with field observations, interviews, and workshops), and social survey (using questionnaires, interviews, and meetings). Spaces for dialogue and collaboration were developed with the community, thereby spawning participatory activities and empowerment. Environmental reordering and reduction of mosquito breeding sites were promoted by: incorporation of environmental issues and vector-borne disease prevention into the school curricula; neighborhood workshops; training of environmental agents to transmit information and train peers; and planning community environmental projects. The households were visited, seeking to integrate the residents into their own community environmental diagnosis. There were significant differences between the pilot areas in knowledge and social practices, but not according to socioeconomic status. The schools and health promotion settings proved to be the main community reference for promoting healthy environmental practices.
\end{abstract}

Dengue; Health Promotion; Local Strategies

\section{Introducción}

La recolonización de Aedes aegypti, vector del dengue y fiebre amarilla urbana en la Argentina siguió patrones similares a lo ocurrido en gran parte del continente americano. Después de algo más de 30 años de la certificación de su erradicación en el país, el vector fue encontrado en la provincia de Misiones en 1986, luego en la provincia de Buenos Aires ${ }^{1}$ y en 1995 en la ciudad de Buenos Aires. Para fines de siglo la distribución geográfica ya era similar a la de los tiempos previos a la erradicación 2 .

Los niveles de infestación de Ae. aegypti en Buenos Aires entre 1997 y 1999 resultaron muy superiores a los registrados durante el programa de erradicación ${ }^{3}$. Entre los posibles factores que facilitaron la recolonización y proliferación vectorial se encuentran la urbanización explosiva que concentra a más 13 millones de personas en una gran metrópolis, las condiciones de mayor favorecimiento climático para el vector ${ }^{4}$, el incremento en la producción o uso doméstico de productos descartables cuyos recipientes o envases pueden funcionar como criaderos y las deficiencias en la educación ambiental que permita prevenir la proliferación del vector.

Desde 1998, periódicamente se produjeron brotes epidémicos de dengue en los países limítrofes y en las provincias del norte de la Argentina 5 , donde no existían antecedentes. 
La llegada de virémicos a la región metropolitana de Buenos Aires ${ }^{6}$, donde la población no tiene antecedentes - y por lo tanto tampoco "memoria" inmunológica de la enfermedad - a cualquiera de los cuatro serotipos circulantes, constituyen factores que agravan la situación.

Este escenario producido en el marco de múltiples escenarios de desestabilización, economías regionales frágiles, profundos cambios sociales y políticos, procesos progresivos de diferenciación e inequidad social y debilitamiento de los sistemas sanitarios, se erige como uno de los principales aspectos de vulnerabilidad socioambiental.

El esquema de control vectorial aplicado en los "tiempos de la erradicación" resultaba inviable, ya que era imposible mantener estrategias verticales - ideadas y concebidas desde los niveles centrales de las administraciones y sin ningún tipo de participación comunitaria. Desde las organizaciones que supervisan los problemas de salud de los países de la región se propusieron programas y proyectos que incluyeran una mayor participación comunitaria en todas sus etapas, desde la planificación hasta la evaluación, con modelos teóricos basados en la planificación estratégica y la atención primaria, bajo el nuevo concepto del "bottom-up" 7, donde a la comunidad se le ofrece participar e involucrarse directamente en el control vectorial intradomiciliario y de barrios.

Esas campañas verticales no tuvieron en cuenta la multiplicidad de dimensiones (social, ambiental, económica, etc.). Entre las razones que influyen en la complejidad de esta situación, se destacan la escasa participación comunitaria en la prevención y el control de Ae. aegypti, la debilidad de las estrategias para el cambio de prácticas de los individuos y comunidades, el desorden ambiental que genera fuentes de proliferación vectorial, la frágil continuidad de las actividades preventivas que compiten con la urgencia de otras demandas ambientales o de salud, la escasa coordinación intersectorial y la insuficiente investigación transdisciplinaria para la construcción de herramientas y desarrollo de estrategias adecuadas.

Las primeras campañas masivas de prevención sobre dengue se realizaron en octubre de 1998, tras la primera epidemia. Debido a la improvisación y urgencia divulgaron información confusa, donde se solicitaba a la población tomar "precauciones frente a una enfermedad" que, salvo en pocas zonas del norte argentino, era totalmente desconocida. Las campañas hicieron muy poco hincapié en las características biológicas del Ae. aegypti y en el reconocimiento de los estadios inmaduros (larvas y pupas) de mosquitos, lo cual resulta indispensable para eliminar o manejar adecuadamente los potenciales criaderos.

Paralelamente, si bien se incentivó la articulación entre los subsectores de salud y de educación de distintas administraciones gubernamentales, no se profundizó en la elaboración de programas con alcance curricular, sostenibles y duraderos. Se trabajó en las aulas, con la modalidad de clases magistrales dadas por especialistas, pero sin promover la participación de docentes y alumnos en la construcción de un proyecto.

En base a estos antecedentes, aceptando que la información adecuada es un factor decisivo, pero no suficiente, hemos presupuesto que la apropiación del conocimiento puede favorecer la adopción de prácticas ambientales saludables para ser aplicadas en situaciones reales de prevención de enfermedades. En este sentido, si los miembros de la comunidad pueden comprobar en su entorno cotidiano la información ofrecida y desarrollar medidas ambientales correctivas ajustadas a su propia realidad, los efectos podrían contribuir a reducir la proliferación vectorial local.

La información, el conocimiento y la percepción han sido consideradas como partes de un sistema continuo que tiende a integrarlas conceptualmente en un proceso de modelación de prácticas, hábitos y conductas a escala colectiva, en pos de lograr un empoderamiento comunitario de saberes tendientes a afirmar las acciones de promoción de la salud y la prevención de enfermedades 8 .

El presente trabajo trató de identificar los conocimientos que dispone la población del área metropolitana de Buenos Aires acerca del dengue y su mecanismo de transmisión, determinar en qué jerarquía se ubica este problema de salud entre los problemas ambientales de las zonas piloto e identificar los referentes comunitarios estratégicos para promover la participación comunitaria. Por otra parte, se describen dos procesos de intervención al irrumpir en experiencias relacionadas con prácticas sostenidas de ordenamiento ambiental saludable, con la expectativa de lograr medidas adecuadas de prevención del dengue $\mathrm{u}$ otras enfermedades transmitidas por vectores domiciliarios.

\section{Materiales y métodos}

La experiencia se realizó en dos áreas contiguas de la región metropolitana, la ciudad de Buenos Aires y el municipio de Vicente López, donde habitan respectivamente 2.768 .772 y 274.082 personas 9 . En cada área se seleccionaron dos zonas 
que representan niveles socioeconómicos-ambientales contrastables y heterogéneos.

Las zonas de la ciudad Autónoma de Buenos Aires se separan geográficamente. Una se encuentra en la zona norte, donde habita la mayor parte de la población de clase media y alta y cuenta con todos los servicios básicos (luz, agua, recolección de basura, acceso a la salud y educación pública y privada). La otra, al sur de la ciudad, agrupa la mayor parte de los asentamientos de escasos recursos donde los servicios de agua potable, recolección de basura y el acceso a la educación y salud constituyen recursos restringidos. En la zona norte se seleccionó el barrio de Villa Pueyrredón, de casas bajas con abundante vegetación y calles arboladas. Este barrio forma parte de la región administrativa CGP12 (Centro de Gestión y Participación) donde habitan 104.229 hombres y 122.084 mujeres según el censo de 2001 en una superficie de $16,3 \mathrm{~km}^{2}$ y una densidad de 13.884 habitantes $/ \mathrm{km}^{2}$. Entre los 76.026 hogares, el $62,8 \%$ son viviendas familiares y el $13,5 \%$ son multifamiliares. En la segunda zona se encuentra el barrio San Martín o Charrúa, una comunidad de familias mayoritariamente de origen boliviano, ubicada dentro del área del barrio de Villa Soldati. Las viviendas son pequeñas y sin patios ni jardines, agrupadas en dos, tres y hasta cuatro plantas a lo largo de pasillos de unos 30 metros de largo. Los servicios de agua y recolección de basura son deficientes. En el CGP5 donde se encuentra este pequeño barrio habitan 64.330 hombres y 70.986 mujeres, en una superficie de $12,5 \mathrm{~km}^{2}$ y una densidad de 10.825 habitantes $/ \mathrm{km}^{2}$. Entre los 38.972 hogares, el $60,7 \%$ son viviendas familiares y el 21,2\% son multifamiliares 10 .

En el caso de Vicente López, administrativamente independiente de la ciudad de Buenos Aires, se trabajó en una porción representativa del sector de clase media y en La Loma, un asentamiento con necesidades básicas insatisfechas, como indicadores de pobreza, con un total de 2.200 habitantes en viviendas ubicadas en fracciones de 6 manzanas, ocupando espacios de fábricas o depósitos abandonados. Sus viviendas no disponen del servicio de agua corriente y cloacas, la energía eléctrica se obtiene mediante conexiones clandestinas y la recolección de la basura se concentra en contenedores de residuos ubicados a la entrada de los asentamientos. Los espacios verdes no existen o son escasos. El acceso a la salud es a través de centros de salud dependientes de la Dirección de Atención Primaria y existe un sólo hospital zonal. La zona de clase media, correspondió a 16 manzanas del barrio de Olivos. Son casas estructuradas con jardín y no se encuentran edificios elevados. Ambas zonas poseen un Centro de Atención Primaria de la Salud, el cual congrega a varios de sus vecinos, escuelas públicas y privadas, y centros deportivos y de recreación.

De acuerdo a las etapas del trabajo, se aplicaron distintas metodologías, apuntando la triangulación de métodos cualitativos y cuantitativos 11, ambos basados en los conceptos de la promoción de la salud y la gestión asociada. Dentro de la promoción de la salud se buscó la acción participativa y el empoderamiento de los actores sociales y/o de los socios - según la denominación utilizada en la metodología del Mapeo de Alcances para programas de salud - (organismos de la salud, educación, ambiente, etc.).

Para las metodologías cualitativas se aplicaron las técnicas de observación participante 12 y entrevistas a los referentes comunitarios, para recolectar información, construir una aproximación diagnóstica comunitaria e identificar prioridades de la comunidad vinculadas a la salud ambiental.

Como parte de la etapa cuantitativa, se realizaron encuestas de modo censal con un cuestionario semi-estructurado a todos los vecinos de las zonas que accedieron a la misma.

Luego se identificaron referentes barriales y gubernamentales como parte de la estrategia. Una vez detectados los sectores interesados en participar - a través del diagnóstico social - se fijaron las acciones a realizar, dependiendo de las características locales, donde se aplicaron los conceptos de promoción de salud basados en dos niveles de intervención, la individual y la poblacional o colectiva buscando su interconexión. Se buscó tener en cuenta el ámbito social y estructural donde se plantean las acciones para modificar los ambientes y para influir en las causas o determinantes de las condiciones de vida - salud 8 . Esto significó tener en cuenta durante todo el trabajo, las condiciones sociales de los individuos y los condicionantes que, como grupos sociales, tienen para actuar y producir cambios en el medio ambiente. Es por ello que se buscó dinamizar acciones sociopolíticas 8 que involucren actores que trasciendan al sector salud y cuya inclusión faciliten experiencias más integradoras y multisectoriales.

Para lograr estos objetivos, en una primera etapa diagnóstica se realizaron reuniones con los distintos actores sociales (organismos gubernamentales locales - centros de salud comunitaria, centros de gestión y participación, instituciones educativas, etc.) y organizaciones no gubernamentales (organizaciones en los barrios, centros de jubilados, etc.)

Posteriormente, se realizaron talleres y encuestas con preguntas abiertas donde se indagó sobre las problemáticas ambientales que más 
preocupaban sus habitantes. Dentro de las mismas se trató de ubicar el orden de prioridad de los mosquitos y las enfermedades que pueden transmitir.

En una tercera etapa se realizaron encuestas y relevamientos entomológicos domiciliarios. En todos los casos, se accedió a las viviendas cuando existió consentimiento de sus propietarios.

En cada oportunidad y antes de entrar, se realizaron las siguientes preguntas (a) $i$ Sabe usted si los mosquitos transmiten enfermedades?, ¿cuáles?; (b) ¿Sabe dónde crían?, ¿podría describir esos sitios?; (c) ¿Cómo se cuida para evitar ser picado por los mosquitos? En ningún caso se tomaron datos socio-demográficos. Posteriormente, se propuso realizar una evaluación entomológica del domicilio. En caso de hallarse larvas de mosquitos se las mostraba y se explicaban las medidas ambientales adecuadas para prevenir su proliferación.

Simultáneamente, en todas las etapas se buscó la asociación con diferentes tipos de actores sociales o socioestratégicos que pudieran facilitar y multiplicar la temática en la comunidad. Para ello se implementaron diferentes estrategias conforme las características de las organizaciones, grupos de cada localidad y los objetivos de cada etapa. Para el caso particular de las instituciones educativas se propuso incorporar temas relacionados con la salud ambiental y prevención de enfermedades asociadas a los mosquitos presentes en el barrio, el hogar y/o entorno en los proyectos transversales de los currícula. El desafío consistió en que fuera diseñado y desarrollado por los propios docentes maestros con participación de los alumnos, con el asesoramiento del grupo de investigación.

Con los grupos que mostraron interés se elaboraron estrategias de intervención. En este trabajo se resaltan dos casos particulares que demostraron una tendencia al empoderamiento temático.

\section{Resultados}

La descripción de los resultados del presente trabajo se ha dividido en cuatro partes, para facilitar la comprensión de los mismos: en primer lugar, se detallan los socios o actores sociales con los que se interactuó y se relatan las acciones desarrolladas a tal efecto; en segundo término, se presentan los resultados obtenidos durante la obtención y construcción del diagnóstico de situación, tanto social como entomológico; en tercer lugar, se describe la experiencia desarrollada con promotoras de los Centros de Salud en Vicente López; y finalmente, se presentan los re- sultados obtenidos en el trabajo con escuelas de nivel primario en una de las zonas piloto de la ciudad de Buenos Aires.

\section{Acercamiento a los referentes sociales}

En relación a las estrategias desarrolladas para sumar socios y que facilitaran la multiplicación de la temática en los barrios de la ciudad de Buenos Aires, se visitaron 18 diferentes tipos de ONG y organizaciones comunitarias (centros $u$ organizaciones de barrios, jubilados, bibliotecas, centros de salud), de las cuales cuatro respondieron con algún tipo de participación en talleres comunitarios y diferentes actividades informativas. En Vicente López se presentó el proyecto y se entrevistaron organizaciones no gubernamentales: tres de educación (dos escuelas privadas y un centro de apoyo escolar), una recreativa, dos comedores comunitarios, una iglesia. Entre las organizaciones gubernamentales de salud, a la Dirección de Salud Ambiental, la de Epidemiología y la Dirección de Atención Primaria y los Centros de Salud relacionados. Entre los educativos a dos escuelas primarias y tres preescolares. Entre los de acción social a dos comedores comunitarios y un Centro de la Mujer.

La mayoría de los referentes contactados mostraron sensibilidad e interés por la problemática ambiental y el trabajo propuesto. Pero dos sectores decidieron participar activamente en el mismo: en la ciudad de Buenos Aires intervinieron las escuelas del Distrito Escolar relacionado con el barrio de Villa Pueyrredón y en Vicente López participaron los Centros de Salud, a través de las promotoras de Salud Comunitaria.

\section{Diagnóstico de situación}

Los resultados obtenidos por observación participante y las entrevistas mostraron que los temas de seguridad (la violencia, los robos, etc.) resultaron los de mayor preocupación. Entre los temas ambientales, la basura resultó el factor de mayor conflicto. Las plagas (ratas, moscas y cucarachas), fueron mencionadas en segundo y tercer lugar, y los mosquitos fueron mencionados como problema en quinto lugar. Las respuestas relacionadas con el riesgo de transmisión del dengue fueron escasas. Las referencias a otros problemas sociales no fueron tomadas en cuenta por exceder los alcances de este trabajo.

Porcentajes superiores al 50\% de la población encuestada manifestó estar informada respecto a que los mosquitos son capaces de transmitir enfermedades, en tres de las zonas piloto (Villa Pueyrredón y Charrúa casi el $60 \%$, Olivos casi el $80 \%$ ), salvo en La Loma donde sólo el 30,1\% de 
los encuestados manifestaron tener algún conocimiento (Figura 1). Respecto al conocimiento de los sitios de cría de los mosquitos, casi no hubo diferencias y en las cuatro zonas piloto menos del $40 \%$ manifestó conocer los lugares de cría de estos insectos (Figura 2).

Sólo entre 15 y $34 \%$ de los encuestados manifestó reconocer el aspecto de las formas inmaduras de los mosquitos.

En cuanto a si conocían la posibilidad de transmisión de dengue por medio de mosquitos, un $34 \%-37 \%$ dieron respuesta afirmativa en la ciudad de Buenos Aires, mientras que sólo el 10\% reconocieron esta posibilidad en el barrio de Olivos y ninguno de los encuestados en el barrio de La Loma ( $\mathrm{p}<0,05$; Tabla 1).

Respecto a los métodos para evitar ser picados por los mosquitos, los encuestados manifestaron usar principalmente métodos químicos y las medidas preventivas fueron consideradas en mayor proporción en la ciudad de Buenos Aires que en Vicente López (Tabla 2).

Las viviendas examinadas en la ciudad de Buenos Aires mostraron niveles de infestación significativamente superiores $(29,7 \%-38,1 \%)$ a las de Vicente López (4,9\%-9,3\%; p < 0,05; Tabla 3) y no se registraron diferencias significativas entre niveles socioeconómicos de cada zona.
Aunque la proporción de criaderos de $A e$. $a e$ gypti entre los recipientes hallados con agua fueron también superiores en Buenos Aires (12,1\%$13,8 \%)$, respecto a Vicente López (4,6\%-9,3\%), las diferencias no resultaron significativas $(p>0,05)$.

\section{Experiencia de intervención con las promotorasde los centros de salud}

Uno de los nexos con la comunidad se realizó a través de las Promotoras de Salud Comunitaria, mujeres que contaban con entrenamiento y cursos previos vinculados a la salud comunitaria por períodos comprendidos entre 2 y 10 años. El equipo de investigación concurrió semanalmente a las reuniones preestablecidas por las promotoras, donde habitualmente se discuten y planifican actividades relacionadas con cuestiones de salud-enfermedad de las áreas programáticas. Desde ese espacio, en algunos casos con apoyo y acompañamiento de los directores de los centros de salud se inició un trabajo de capacitación vinculado a la temática del ordenamiento ambiental domiciliario, incluyendo la prevención del vector del dengue. Las propias necesidades y realidades dispusieron tiempos y momentos diferenciados para cada grupo de trabajo. Los espacios de capacitación generaron distintas formas de difusión

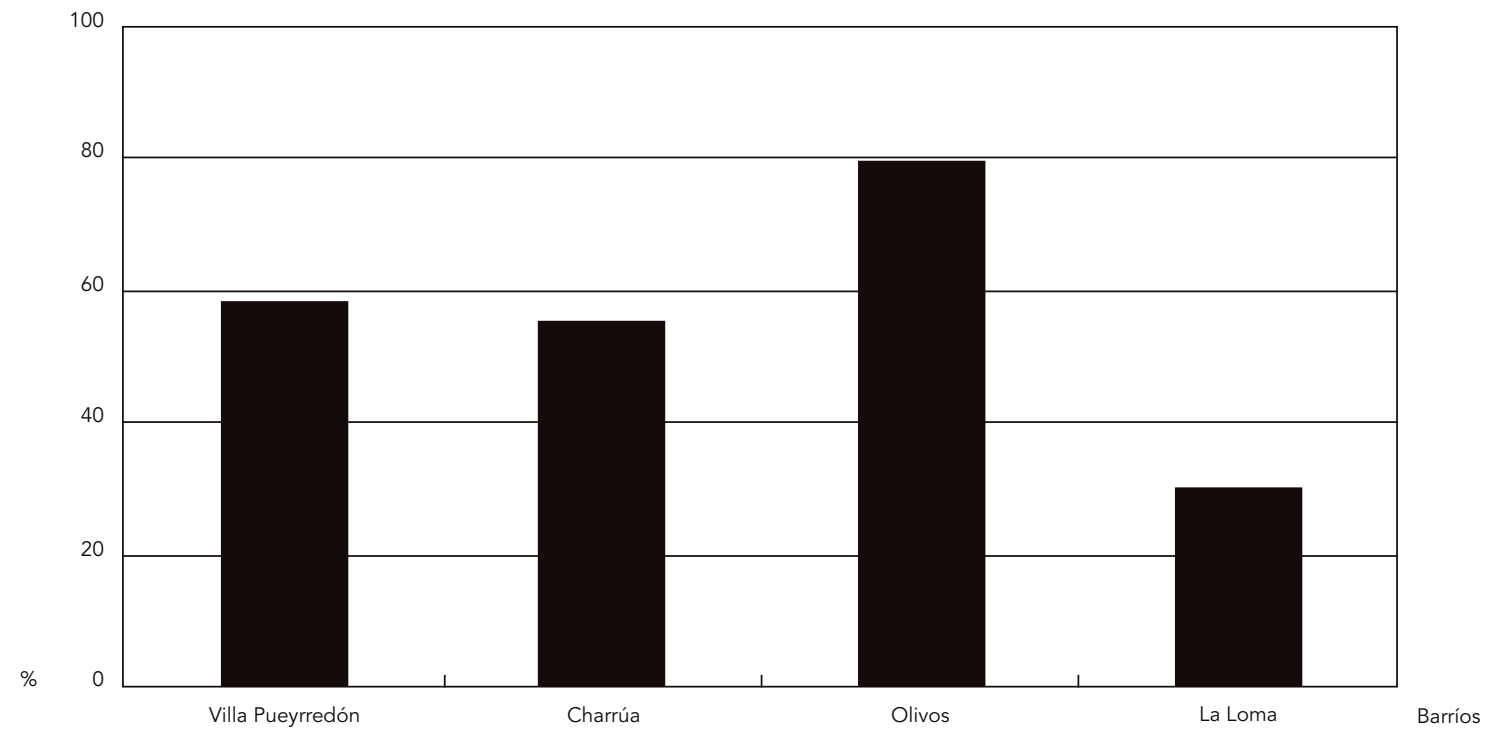


Figura 2

Encuestas domiciliarias realizadas en la ciudad de Buenos Aires (Villa Pueyrredón y Barrio Charrúa) y en Vicente López (La Loma y Olivos), Argentina, sobre donde crían los mosquitos en los domicilios.

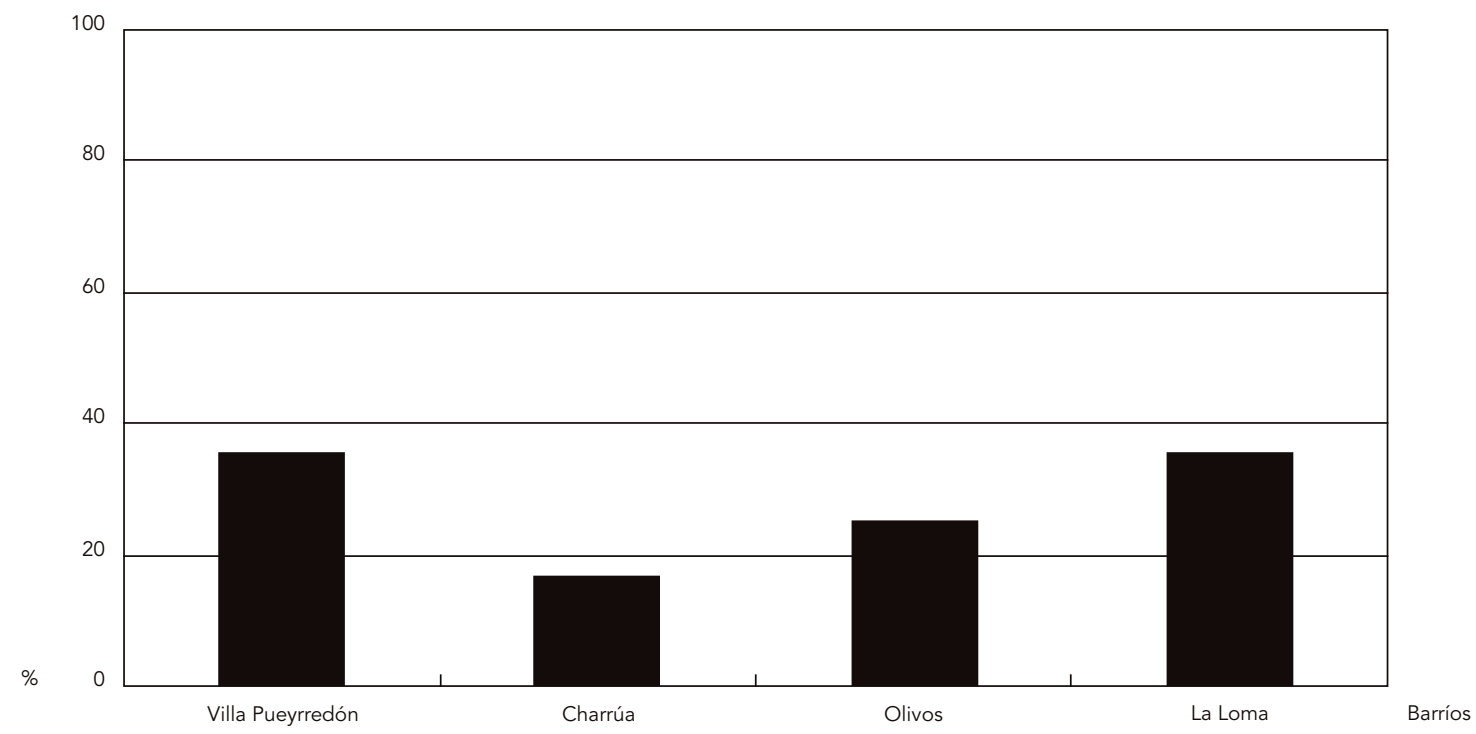

Tabla 1

Enfermedades que transmiten los mosquitos según los encuestados de Buenos Aires (Villa Pueyrredón y Barrio Charrúa) y Vicente López (La Loma y Olivos), Argentina.

\begin{tabular}{|c|c|c|c|c|}
\hline & \multicolumn{2}{|c|}{ Buenos Aires } & \multicolumn{2}{|c|}{ Vicente López } \\
\hline & $\begin{array}{c}\text { Villa } \\
\text { Pueyrredón } \\
\text { (\%) }\end{array}$ & $\begin{array}{c}\text { Charrúa } \\
\text { (\%) }\end{array}$ & $\begin{array}{c}\text { Olivos } \\
\text { (\%) }\end{array}$ & $\begin{array}{c}\text { La Loma } \\
\text { (\%) }\end{array}$ \\
\hline Dengue & 34,8 & 37,1 & 10,0 & 0,0 \\
\hline Otras (malaria, encefalitis, filariasis, fiebre amarilla) & 8,7 & 4,3 & 70,0 & 30,1 \\
\hline Otras (no necesariamente correctas) & 15,2 & 17,1 & & \\
\hline Total encuestas & 46,0 & 70,0 & 30,0 & 72,0 \\
\hline
\end{tabular}

Nota: se permitió más de una respuesta.

de prácticas saludables en cada lugar (obras de títeres, obra de teatro, muestras durante los relevamientos entomológicos).

Durante la evaluación anual del proceso, las promotoras propusieron profundizar la capacitación sobre ordenamiento ambiental a través de un curso, y hacerla extensiva a los equipos de los restantes 18 centros de salud del municipio. El diseño y desarrollo del mismo se realizó de manera participativa. En una primera instancia se proyectaron fotografías de entornos domiciliarios típicos, donde los participantes identificaron los factores ambientales relevantes que podrían afectar la salud. Los químicos (presencia de plomo, asbesto, clorados, fosforados, etc.), la radiación electromagnética y los biológicos contaminantes (bacterias en aguas servidas, hongos, etc.) y plagas (ratas, piojos, ácaros, moscas y mosquitos), dando especial énfasis a aquellos que favorecen la proliferación del vector del dengue. En 
Métodos mencionados por los encuestados de Buenos Aires (Villa Pueyrredón y Barrio Charrúa) y Vicente López (La Loma y Olivos), Argentina, para evitar ser picados por los mosquitos.

\begin{tabular}{lcccc}
\hline & \multicolumn{2}{c}{ Buenos Aires } & \multicolumn{2}{c}{ Vicente López } \\
& Villa Pueyrredón (\%) & Charrúa (\%) & Olivos (\%) & La Loma (\%) \\
\hline Métodos químicos & 39,1 & 20,0 & 55,1 & 64,4 \\
Métodos mecánicos & 0,0 & 2,9 & 6,4 & 1,4 \\
Medidas preventivas & 39,1 & 45,7 & 3,8 & 0,0 \\
Total encuestas & 46,0 & 70,0 & 78,0 & 73,0 \\
\hline
\end{tabular}

Nota: las respuestas fueron agrupadas en tres categorías.

Tabla 3

Viviendas infestadas y recipientes con agua que servían de criaderos de Ae. aegypti en Buenos Aires (Villa Pueyrredón y Barrio Charrúa) y Vicente López (La Loma y Olivos), Argentina.

\begin{tabular}{lcccc}
\hline & \multicolumn{2}{c}{ Buenos Aires } & \multicolumn{2}{c}{ Vicente López } \\
Zona & Villa Pueyrredón (\%) & Charrúa (\%) & Olivos (\%) & La Loma (\%) \\
\hline \multirow{2}{*}{$\begin{array}{l}\text { Viviendas } \\
\text { Criaderos }\end{array}$} & 38,1 & 29,7 & 9,3 & 4,9 \\
\hline
\end{tabular}

Nota: las respuestas fueron agrupadas en tres categorías.

una segunda etapa los participantes identificaron con apoyo fotográfico los temas relevantes de preocupación sobre sus barrios, dichos temas se transformaron en el eje central del curso. La participación de una profesional de uno de los Centros de Salud como parte del equipo docente, sirvió para transformar el reclamo y atribución de la responsabilidad ambiental hacia las autoridades gubernamentales en una apropiación temática y una responsabilidad individual/grupal del cuidado y manejo del entorno. En la etapa de cierre del curso, los 45 participantes presentaron seis proyectos comunitarios sobre ordenamiento ambiental por localidades. Los referentes de los proyectos, interesados por la ejecución de sus propuestas, buscaron de manera autónoma la modalidad de financiación, entre los cuales uno obtuvo financiación de una fundación para su ejecución en el barrio y otro firmó un convenio con la Universidad Nacional de Cine, para la realización de un video informativo de divulgación escolar. El conjunto de las experiencias sirvió para que las promotoras pudieran identificarse como parte de una comunidad capaz de promover su propio cambio.

\section{Experiencias de intervención en las escuelas}

Durante 2005 se establecieron contactos con las supervisiones de los Distritos Escolares no. 16 y 19, correspondientes a las áreas de trabajo en los barrios Charrúa y Villa Pueyrredón, respectivamente. Sólo los directivos de tres escuelas de Villa Pueyrredón decidieron participary se articularon encuestas y entrevistas a docentes de distintas asignaturas. De ellas, una escuela logró trabajar transversalmente, esto es que distintas materias abordaran el mismo tema desde ópticas diferentes. Se realizaron talleres y clases ilustrativas con docentes y alumnos. Una de las maestras se asoció al equipo de investigación y promovió la idea con entusiasmo entre sus colegas. Entre los productos resultantes, los alumnos produjeron afiches, folletos, reportajes y un cortometraje dirigido a los padres de los alumnos. Los resultados se expusieron en actos escolares de fin de año.

Durante el transcurso del trabajo se registró que la información presente en los manuales de texto escolares sobre el ciclo de vida de los mosquitos y el riesgo de transmitir enfermedades no existe, es escasa o contiene importantes errores conceptuales (ej. gráficos donde se asocia el ciclo 
del Aedes. sp. con charcos temporarios o represas, sin mostrar la importancia de la proliferación en las viviendas).

Los resultados obtenidos en el primer año produjeron interés y apropiación del proyecto por parte del supervisor del Distrito Escolar no. 16. Esta situación no se repitió en el caso del otro distrito por cuestiones de inseguridad y violencia que generaron continuos cambios de autoridades, licencias o traslados de los docentes.

Durante 2006, se formalizó la asociación estratégica con la supervisión del Distrito Escolar no. 16 y se llevó a cabo la selección de siete escuelas, las cuales participaron en forma articulada con ese nivel jerárquico de distritos.

Se trabajó, primero, con los niveles de conducción institucional - directoras, vicedirectoras y secretarias - a través de la coordinación y planificación de diferentes estrategias de abordaje, construyendo alternativas específicas para la realidad de cada escuela, grado o docente.)

En una segunda etapa se trabajó con los docentes seleccionados. Se llevaron a cabo entrevistas informales (semanales o quincenales) con cada uno de ellos y se les entregó material teórico-práctico. Las diferentes actividades se planificaron y coordinaron de forma consensuada. Se realizaron asesoramientos y se sugirieron posibles actividades y un seguimiento o acompañamiento posterior del proceso. Se trabajó en diferentes ámbitos dentro (aula, biblioteca, laboratorio de ciencias, laboratorio de informática) y fuera de la escuela (viviendas de alumnos y docentes, comercios, vía publica y laboratorios de la Universidad). Los docentes interactuaron trasladando la temática a sus pares de otros grados, e incluso de otras escuelas, docentes de otras disciplinas y otros actores de la comunidad escolar. Se interactuó con docentes de áreas tan disímiles como: plástica, teatro, música, informática, publicidad, matemática, prácticas del lenguaje, bibliotecario, ayudante de clases prácticas, etc. (Tabla 4).

Se realizaron distintos tipos de actividades, de las cuales la mayoría fueron propuestas y planificadas por los propios docentes y alumnos, donde participaron en forma directa más de 200 alumnos. Entre las actividades de terreno se realizaron encuestas a familiares, vecinos o transeúntes que pasaban por el barrio, búsquedas de formas inmaduras de mosquitos en sus hogares, colocación de ovitrampas fabricadas por los propios alumnos en aproximadamente 75 viviendas. En una de las escuelas, los alumnos establecieron contacto por correo con sus pares de una escuela de Salta, provincia limítrofe con Bolivia, donde existe transmisión del dengue y registraron que los alumnos de esas localidades no contaban con información sobre la enfermedad y las medidas de prevención adecuadas.

Entre las actividades intra-escolares se realizaron talleres interactivos con participación de docentes, alumnos y especialistas; entre las producciones obtenidas cabe destacar la realización de canciones y danzas, obras de títeres y de teatro donde se mostraban caricaturizaciones de cómo respondía la población a sus encuestas o al desconocimiento respecto a un vector que se encontraba en sus viviendas.

También se realizaron actividades ilustrativas como maquetas, juegos didácticos; presentaciones audiovisuales, utilizando programas de computación y multimedia, afiches y folletos informativos preventivos, así como charlas realizadas por los propios alumnos en otros cursos, artículos periodísticos en revistas y/o periódicos escolares; presentaciones a la comunidad como clases abiertas con familiares y un vídeo realizado por los docentes y alumnos, el cual fue proyectado a los familiares a través de reuniones abiertas y a otras escuelas.

La actividad culminó con un encuentro de distritos en un teatro municipal. Participaron todos los docentes y alumnos, e intercambiaron sus experiencias y resultados, donde mostraron sus producciones y realizaron en forma conjunta una evaluación del proyecto. Dichos resultados se publicaron en un documento. Esta actividad contó con la presencia de autoridades centrales del sistema de salud y del de educación de la ciudad de Buenos Aires.

Por otra parte, es de destacar también que los conocimientos previos sobre el tema, entre los educadores también eran escasos. Por ejemplo, sólo dos de siete directores de escuela de un distrito escolar conocían previamente como eran las larvas de los mosquitos. Durante una reunión realizada con los mismos directores y el supervisor, antes del inicio del ciclo lectivo del año siguiente se observó un importante cambio en los saberes adquiridos y un marcado interés por continuar con las actividades.

\section{Discusión}

A raíz de las primeras epidemias a fines del milenio en el norte de Argentina y la difusión oficial sobre las prácticas preventivas, cierto grado de conocimiento de la enfermedad se había instalado en la sociedad, como lo muestran los resultados de las encuestas realizadas en este trabajo; sin embargo, el riesgo de transmisión debido a la presencia de criaderos en los hogares demuestra una importante ruptura entre el conocimiento, el manejo de la información por parte de la 
Acciones desarrolladas por cada una de las escuelas del Distrito Escolar no.16 de la ciudad de Buenos Aires, Argentina, involucradas en el trabajo.

\begin{tabular}{|c|c|c|c|c|c|c|}
\hline \multicolumn{4}{|c|}{ Participación escolar } & \multicolumn{3}{|c|}{ Participación extraescolar } \\
\hline Escuela & Clase & Público & Contenidos & Transversalidad & Materiales & Contactos \\
\hline 6 & 6o & Familiares, vecinos & $\begin{array}{c}\text { Dengue, su } \\
\text { transmisión y } \\
\text { prevención; criaderos } \\
\text { en domicilios }\end{array}$ & $\begin{array}{l}\text { Naturales; oral; } \\
\text { sociales; música; } \\
\text { informática }\end{array}$ & $\begin{array}{l}\text { Afiches y folletos; } \\
\text { publicidad radial y } \\
\text { gráfica }\end{array}$ & $\begin{array}{l}\text { Consultaron a la } \\
\text { comunidad }\end{array}$ \\
\hline 12 & 6으 & $\begin{array}{l}\text { Contactos en vía } \\
\text { pública y parque }\end{array}$ & $\begin{array}{l}\text { Enfermedades } \\
\text { vectoriales, } \\
\text { transmisión y } \\
\text { prevención; } \\
\text { responsabilidad } \\
\text { individual, familiar y } \\
\text { comunitaria }\end{array}$ & $\begin{array}{c}\text { Naturales; oral; } \\
\text { informática; sociales }\end{array}$ & $\begin{array}{c}\text { Afiches y folletos } \\
\text { de distribución por } \\
\text { barrios }\end{array}$ & $\begin{array}{c}\text { Trabajo con familias } \\
\text { y vecinos }\end{array}$ \\
\hline 13 & 6o & Familiares, vecinos & $\begin{array}{l}\text { Biología del } \\
\text { vector, lugares de } \\
\text { cría, transmisión. } \\
\text { Métodos de control } \\
\text { y prevención; sitios } \\
\text { de cría }\end{array}$ & $\begin{array}{l}\text { Plástica; tecnología; } \\
\text { oral; artesanal; } \\
\text { música }\end{array}$ & $\begin{array}{c}\text { Folletos, } \\
\text { entrevistas; títeres, } \\
\text { dramatizaciones; } \\
\text { láminas, mapas, } \\
\text { juegos, crucigramas }\end{array}$ & $\begin{array}{c}\text { Participación familiar } \\
\text { durante reuniones } \\
\text { pedagógicas }\end{array}$ \\
\hline 14 & 4으, 5으, 6으 & Familiares, vecinos & $\begin{array}{l}\text { Biología del vector; } \\
\text { riesgos; métodos } \\
\text { de protección; sitios } \\
\text { de cría }\end{array}$ & $\begin{array}{c}\text { Naturales; oral; } \\
\text { sociales; plástica; } \\
\text { matemática; } \\
\text { informática; artesanal } \\
\text { y técnica }\end{array}$ & $\begin{array}{l}\text { Grafitos, afiches, } \\
\text { volantes, folletos }\end{array}$ & $\begin{array}{c}\text { Armado y colocación } \\
\text { de ovitrampas. } \\
\text { Intercambio postal } \\
\text { con alumnos de la } \\
\text { provincia de Salta }\end{array}$ \\
\hline 17 & 60 & Familiares, vecinos & $\begin{array}{l}\text { Biología del } \\
\text { vector; modos } \\
\text { de transmisión; } \\
\text { métodos de } \\
\text { prevención; sitios } \\
\text { de cría }\end{array}$ & Informática; música & $\begin{array}{l}\text { Dramatización; } \\
\text { murga, afiches, } \\
\text { murales }\end{array}$ & $\begin{array}{l}\text { Encuesta pre - y post } \\
\text { - acciones educativas }\end{array}$ \\
\hline 22 & 4으, 5으, 6으, 7으 & $\begin{array}{c}\text { Encuesta, clase } \\
\text { abierta }\end{array}$ & $\begin{array}{l}\text { Biología del } \\
\text { vector; modos } \\
\text { de transmisión; } \\
\text { métodos de } \\
\text { prevención; sitios de } \\
\text { cría-enfermedad }\end{array}$ & $\begin{array}{c}\text { Informática; } \\
\text { oral; naturales; } \\
\text { plástica; sociales; } \\
\text { matemáticas }\end{array}$ & $\begin{array}{c}\text { Afiches, ovitrampas, } \\
\text { volantes, puestos, } \\
\text { maquetas }\end{array}$ & $\begin{array}{c}\text { Preparación y } \\
\text { colocación de } \\
\text { ovitrampas; difusión } \\
\text { con un puesto en } \\
\text { una plaza del barrio }\end{array}$ \\
\hline 24 & 60 & $\begin{array}{l}\text { Encuesta, vídeo, } \\
\text { investigación } \\
\text { bibliográfica }\end{array}$ & $\begin{array}{l}\text { Biología del } \\
\text { vector; modos } \\
\text { de transmisión; } \\
\text { métodos de } \\
\text { prevención }\end{array}$ & $\begin{array}{l}\text { Sociales; naturales; } \\
\text { oral; plástica; música }\end{array}$ & $\begin{array}{c}\text { Afiches, volantes, } \\
\text { vídeos, canciones, } \\
\text { coreografía }\end{array}$ & $\begin{array}{l}\text { Transferencia } \\
\text { del vídeo a otras } \\
\text { escuelas y a una } \\
\text { Organización no } \\
\text { Gubernamental; } \\
\text { muestra fotográfica }\end{array}$ \\
\hline
\end{tabular}


población y la implementación práctica de estos saberes. Resultados similares fueron registrados en Cuba 13 y Puerto Rico 14, quienes pudieron distinguir una clara diferencia entre los conocimientos, las actitudes y las prácticas vinculadas al dengue y su transmisión.

Entre las posibles explicaciones a estos resultados se podría tener en cuenta la preocupación de las comunidades por otro tipo de problemas sociales, como la drogadicción, la violencia u otros problemas ambientales de mayor peso que los criaderos de mosquitos, como, por ejemplo, los permanentes conflictos por la disposición y recolección de la basura domiciliaria. Si bien existe difusión oficial sobre un cierto riesgo epidemiológico de un brote de dengue en la región metropolitana de Buenos Aires, la ausencia de transmisión viral autóctona y la virtual invisibilidad de los criaderos de estos mosquitos en las propias viviendas, inhiben una toma de conciencia y responsabilidad ambiental para generar prácticas que sirvan para mejorar la calidad ambiental en los domicilios.

Sin embargo, las experiencias de intervención llevadas a cabo durante el presente trabajo demostraron que en algunos sectores de nuestra sociedad existe avidez por el conocimiento, donde es posible diseñar estrategias propias y llevar a cabo prácticas que puedan sostenerse y enriquecerse por sí mismas, fortaleciendo la propuesta el sentido que la adquisición de conocimientos sea considerada una complejidad socioambiental y biológica; pudiendo producir entre determinados referentes sociales un empoderamiento con suficiente entusiasmo como para contagiar a otros sectores de las distintas redes que componen a una sociedad

En una sociedad altamente fragmentada como la que presentaba la Argentina en el momento de desarrollo del presente trabajo, los Centros de Salud y las escuelas son entornos naturales respetados por el conjunto de la comunidad.

Los centros de atención primaria se ocupan de las distintas campañas relacionadas con el proceso de salud-enfermedad-atención. La incorporación de conceptos biológicos-ambientales básicos, como parte de un entorno saludable, sirve para sensibilizar y potenciar sus capacidades humanas hacia saltos cualitativos en una dimensión más englobadora (ambiente-salud). Los cursos teórico-prácticos donde se recupera la mirada de los/as participantes, llevando el plano de lo teórico al plano de lo cotidiano, de la práctica de los propios grupos resultó de gran utilidad para generar empoderamiento temático $15,16,17$. En esta experiencia, el grupo de promotoras de salud favoreció el inicio de un proceso en términos de participación social en salud. Con el apoyo de espacios preexistentes se visualizó la potencialidad de fortalecer la organización social "desde abajo", al promoverse el empoderamiento temático para procesos participativos comunitarios con protagonismo ciudadano. Por el contrario, los trabajos generados verticalmente a través de los funcionarios, mostraron el riesgo de paralizar los proyectos por la volatilidad de sus cargos. El interés y la apropiación de los espacios se vieron garantizados por la escucha de las demandas y por la inclusión de la población destinataria en todo el proceso, para dar cuenta de una "real" participación comunitaria.

El caso de las escuelas de Villa Pueyrredón demostró que los maestros de la región no cuentan con material adecuado para enseñar temas ambientales relacionados con la salud. Esto concuerda con los resultados de las encuestas realizadas sobre la población y además, podría extrapolarse a los tomadores de decisiones en cuestiones de salud, ya que son parte de la misma sociedad. Los resultados sugieren que es necesario rever los contenidos de los manuales que usan los alumnos y docentes y sugerir la incorporación de capítulos relacionados con el ambiente domiciliario saludable.

El desarrollo de actividades de investigación del entorno domiciliario y/o barrial desde la transversalidad de las distintas asignaturas ha servido para que los alumnos adopten una mirada innovadora sobre el ambiente que habitan (estudios en sus viviendas), la biología de un vector (capaz de transmitir distintas enfermedades), la sociedad con la que conviven (resultados de las encuestas y teatralizaciones), significado de los números (encuestas, resultados de ovitrampas o recipientes con agua), incorporando naturalmente la complejidad de sistemas ecológicos a tener en cuenta para prevenir enfermedades. Por otro lado, la experiencia lograda por los docentes con los proyectos de su propia autoría y el asesoramiento del grupo de investigación ha servido para lograr entusiasmo y un empoderamiento temático, capaz de generar motivación entre los alumnos y sus familiares. El maestro dispone así de una herramienta, que sirve para construir conocimiento y generar nuevas inquietudes, al lograr el interés de sus alumnos por un tema que aparece con una múltiple invisibilidad: la presencia en sus propias viviendas de criaderos desconocidos de un mosquito capaz de transmitir una enfermedad que aún no reconoce casos locales de la misma y que forma parte de una realidad ambiental. Asimismo, el ejercicio intelectual de los niños, encuentra una ejercitación concreta que se traduce en nuevas prácticas de salud y cuidado del medio ambiente. Poniendo en evidencia que las actividades educativas, diri- 
gidas al individuo y que tienden a un cambio en su comportamiento, no pueden estar separadas del marco de políticas públicas saludables y de procesos participativos.

Este modelo se torna novedoso por su construcción teórico-metodológica, que permite sugerir líneas de acción en salud y educación pública en espacios formales y no formales, que promuevan igualdad de conocimientos y empoderamiento comunitario para generar prácticas saludables relacionadas con la prevención de la proliferación del vector antes de que suceda la enfermedad.

\section{Resumen}

Con objetivos de prevención de dengue, promoción de la salud e investigación-acción se desarrollaron dos experiencias en zonas piloto de Buenos Aires y Vicente López. En cada zona se identificaron referentes comunitarios, gubernamentales y no gubernamentales. Se realizó un diagnóstico entomológico (mediante ovitrampas), ambiental (con observaciones en terreno, encuestas y talleres), y social (utilizando cuestionarios, entrevistas y reuniones). Se construyeron espacios de diálogo y trabajo conjunto con la comunidad, generando acciones participativas y empoderamiento. Se fomentó el ordenamiento ambiental y la reducción de criaderos de mosquitos a través de incorporación de temas ambientales y prevención de enfermedades vectoriales a los currícula escolares; talleres vecinales de difusión; capacitación de promotoras ambientales para transmitir información y para capacitar a pares; planificación de proyectos ambientales de la comunidad. Se entró en los domicilio, buscando integrar a sus habitantes en el diagnóstico ambiental propio y comunitario. Se registraron diferencias significativas entre conocimientos y prácticas sociales, para ambas zonas piloto, pero no por nivel socioeconómico. Las escuelas y los entornos de promoción de salud resultaron ser los principales referentes comunitarios para fomentar prácticas ambientales saludables.

Dengue; Promoción de la Salud; Estrategias Locales

\section{Colaboradores}

El artículo se redactó a partir de la información colectada por los autores del proyecto y la dirección de investigadores N. Schweigmann, A. Rizzotti, G. Castiglia, F. Gribaudo y E. Marcos redactor principal del artículo. N. Burroni responsable de las encuestas entomológicas y colaboradora en las encuestas sociales en la Ciudad de Buenos Aires que figuran en el trabajo. G. Freire responsable de las encuestas entomológicas, colaboradora en las encuestas sociales y aportó información y colaboró en la revisión crítica del trabajo. V. D’Onofrio responsable de las encuestas las encuestas sociales aportó información y colaboró en la revisión crítica del trabajo. S. Oberlander participó activamente en las encuestas sociales y en la interacción con las escuelas de la ciudad de Buenos Aires que figuran en el trabajo. H. Schillaci participó activamente en las encuestas sociales Aportó información y colaboró en la revisión crítica del trabajo. S. Gómez colaboradora de las encuestas entomológicas y sociales que figuran en el trabajo. S. Maldonado aportó información mediante las encuestas sociales C. Serrano aportó información mediante las encuestas sociales y entomológicas y colaboró con una visión crítica en los resultados que se presentan en el artículo.

\section{Agradecimientos}

Grupo de promotoras de Vicente López; directores, maestros, alumnos y supervisor del Distrito Escolar no. 16 de la ciudad de Buenos Aires. Proyecto financiado por International Development Research Centre - Canadá 101814-002. 


\section{Referencias}

1. Campos R. Presencia de Aedes (Stegomyia) aegypti (L.) (Diptera: Culicidae) en la localidad de Quilmes (Buenos Aires, Argentina). Rev Soc Entomol Argent 1993; 52:36.

2. Curto SI, Boffi R, Carbajo AE, Plastina R, Schweigmann N. Reinfestación del territorio Argentino por Aedes aegypti. Distribución geográfica (19941999). In: Salomón DS, editor. Actualizaciones en artropodología sanitaria Argentina. Buenos Aires: Fundación Mundo Sano; 2002. p. 127-37.

3. Schweigmann N, Orellano P, Kuruc J, Vera MT, Vezzani D, Méndez A. Distribución y abundancia de Aedes aegypti (Diptera: Culicidae) en la ciudad de Buenos Aires. In: Salomón DS, editor. Actualizaciones en artropodología sanitaria Argentina. Buenos Aires: Fundación Mundo Sano; 2002. p. 155-60.

4. Garín AB, Bejarán R, Carbajo AE, Casas SC, Schweigmann N. Atmospheric control of Aedes aegypti populations in Buenos Aires (Argentina) and its variability. Int J Biometeorol 2000; 44:148-56.

5. Aviles G, Rangeón G, Vorndam V, Briones A, Baroni A, Enria A, et al. Dengue reemergence in Argentina. Emerg Infect Dis 1999; 5:575-8.

6. Seijo A, Curcio D, Aviles G, Cernigoi B, Deodato B, Lloveras S. Imported dengue in Buenos Aires, Argentina. Emerg Infect Dis 2000; 6:655-6.

7. Spiegel J, Bennett S, Hattersley L, Hayden MH, Kittayapong P, Nalim S, et al. Barriers and bridges to prevention and control of dengue: the need for a socialecological approach. EcoHealth 2005; 2:273-90.

8. Restrepo H, Málaga H. Promoción de la salud: cómo construir vida saludable. Bogotá: Editorial Médica Panamericana; 2001.

9. Instituto Nacional de Estadísticas y Censos. Censo nacional 2001. Buenos Aires: Instituto Nacional de Estadísticas y Censos; 2001.
10. Dirección General de Estadísticas y Censos, Secretaría de Hacienda y Finanzas. Anuario estadístico 2004, Ciudad de Buenos Aires. Tomo 1. Buenos Aires: Gobierno de la Ciudad Autónoma de Buenos Aires; 2005.

11. Taylor SJ, Bogdan R. Introducción a los métodos cualitativos de investigación. Barcelona: Editorial Paidos; 1992.

12. Guber R. El salvaje metropolitano. Buenos Aires: Editorial Paidos; 2004.

13. Espinoza-Gómez F, Hernández-Suárez CM, CollCárdenas R. Factores que modifican los índices larvarios de Aedes aegypti en Colima, México. Rev Panam Salud Pública 2001; 10:6-12.

13. Fajardo P, Monje CA, Lozano G, Realpe O, Hernández LE. Nociones populares sobre "dengue" y "rompehuesos", dos modelos de la enfermedad en Colombia. Rev Panam Salud Pública 2001; 10:161-9.

14. Pérez-Esclarín A. Educación popular y educación formal. In: XXXII Congreso Internacional Antigua. Guatemala: Movimiento de Educación Popular Integral, y Promoción Social, Federación Internacional de Fe y Alegría; 2001. p. 1-17.

15. Vargas L, Bustillos G. Técnicas participativos para la educación popular. Buenos Aires: Editorial Humanitas; 1990.

16. Muguiro-Ibarra F. Trabajo con la comunidad y ciudadanía. In: Educación popular, comunidad y desarrollo sustentable. XXXI Congreso Internacional. Lima: Federación Internacional de Fe y Alegría, Movimiento de Educación Popular Integral y Promoción Social; 2000.

Recibido el 06/Nov/2007

Versión final presentada el 03/Ene/2008

Aprobado el 22/Ene/2008 